\title{
Associação entre supervisão parental e comportamento sedentário e de inatividade física em adolescentes brasileiros
}

\author{
Association between parental supervision and sedentary behavior \\ and physical inactivity and among Brazilian adolescents
}

Clara Pereira Santana (https://orcid.org/0000-0002-6661-9100) ${ }^{1}$

Hiago Alessandro Soares Nunes (https://orcid.org/0000-0001-7042-4085) ${ }^{1}$

Aline Natália Silva (https://orcid.org/0000-0002-1811-3703) ${ }^{2}$

Catarina Machado Azeredo (https://orcid.org/0000-0002-6189-4429) ${ }^{3}$
${ }^{1}$ Graduação em Gestão em Saúde Ambiental, Instituto de Geografia, Universidade Federal de Uberlândia (UFU). Av. Pará 1720, Umuarama. 38405-320 Uberlândia MG Brasil. catarina.azeredo@ufu.br ${ }^{2}$ Programa de PósGraduação em Ciências da Saúde, Faculdade de Medicina, UFU. Uberlândia MG Brasil.

${ }^{3}$ Faculdade de Medicina, UFU. Uberlândia MG Brasil.

\begin{abstract}
The scope of this study was to assess the association between parental supervision and sedentary behavior and physical inactivity among Brazilian adolescents. Data of 102,072 students attending 9 th grade from public and private school gathered in the 2015 Brazilian National Survey of School Health (PeNSE) were used. Adolescents were sedentary and physical inactive when they spent $\geq 3$ hours/day sitting and practiced less then 60min/day of physical activity, respectively. Frequency of parental supervision (never, sometimes, always) was assessed through checking school homework, knowledge about what the adolescents do in their free time and understanding their problems. Logistic regression was applied and duly adjusted for sociodemographic variables. Among the adolescents, $56.3 \%$ were sedentary and $78.1 \%$ were inactive. Checking school homework was associated with lower odds of physical inactivity and sedentary behavior. Knowing what adolescents did in their free time and inspecting their belongings was associated with lower odds of physical inactivity. The understanding of problems by parents/guardians was associated with lower odds of sedentary behavior. The conclusion drawn is that greater parental supervision is associated with less physical inactivity and less sedentary behavior.

Key words Parental supervision, Adolescence, Sedentary behavior, Physical inactivity
\end{abstract}

Resumo Avaliamos associações da supervisão parental com o comportamento sedentário e a inatividade física em adolescentes brasileiros. Utilizamos dados da Pesquisa Nacional de Saúde do Escolar (PeNSE) de 2015, que avaliou 102.072 escolares do $9^{\circ}$ ano. Consideramos sedentários os adolescentes que ficavam $\geq 3$ horas/dia sentados $e$ inativos os que praticaram $<60 \mathrm{~min} /$ dia de atividade física. A supervisão parental foi avaliada pela frequência (nunca, às vezes, sempre) de atividades com verificar o dever, saber sobre o tempo livre e entender os problemas do adolescente. Foram realizadas análises de regressão logística, ajustadas para variáveis sociodemográficas. Dentre os avaliados, $56,3 \%$ eram sedentários e $78,1 \%$ inativos. Verificar atividades escolares se associou à menor odds de inatividade e sedentarismo. Ter ciência do que o adolescente fazia no tempo livre e mexer nas coisas do adolescente se associaram a menor odds de inatividade física. A compreensão de problemas pelos responsáveis se associou à menor odds de sedentarismo. Concluímos que maior supervisão parental se associou a menor sedentarismo $e$ menor inatividade física entre adolescentes.

Palavras-chave Supervisão Parental, Adolescência, Sedentarismo, Inatividade fisica 


\section{Introdução}

A inatividade física e o sedentarismo estão entre os principais fatores de risco para as Doenças Crônicas não Transmissíveis (DCNT) e foram responsáveis por $9 \%$ em 2008 e 3,8\% de 2002 a 2011, respectivamente, das mortes prematuras globalmente ${ }^{1,2}$. O sedentarismo é definido como o tempo dedicado a atividades estáticas com baixo gasto calórico $(\leq 1,5 \mathrm{MET})$ nas posições sentada ou deitada ${ }^{3,4}$. A maioria dos estudos avaliam o sedentarismo em crianças e adolescentes pelo tempo em frente às telas ${ }^{5,6}$. Já a inatividade física compreende a realização de menos de 60 minutos de atividade física diariamente, contabilizando dimensões do lazer, deslocamento e atividades na escola ${ }^{7}$. Os dois comportamentos são cada vez mais comuns na sociedade ${ }^{6}$ e como captam dimensões distintas, uma pessoa pode ser fisicamente ativa e ao mesmo tempo sedentária ${ }^{8}$. Dessa forma, os determinantes da inatividade física e do sedentarismo podem ser diferentes e, portanto, os dois comportamentos devem ser avaliados de forma complementar e não excludente ${ }^{9}$. No Brasil, $71 \%$ e $78 \%$ dos adolescentes foram considerados inativos e sedentários, respectivamen$\mathrm{te}^{10,11}$.

Como a adolescência é um período marcado por mudanças físicas, emocionais e sociais, requer maior atenção familiar e social ${ }^{12}$. Os hábitos familiares e o vínculo dos adolescentes com os pais são fatores que podem determinar comportamentos, ainda que na adolescência a influência dos pares ganhe importância ${ }^{13-15}$. Alguns autores observaram que tanto o sedentarismo como a inatividade física dos adolescentes podem ser minimizados através de monitoria positiva e acompanhamento de suas rotinas por seus supervisores parentais ${ }^{16-18}$.

O interesse pelo papel da família, nos níveis de atividade física e sedentarismo do adolescente, ganhou destaque no meio acadêmico nos últimos anos ${ }^{19,20}$. Fatores como imposição de normas e limites, suporte dos pais para a superação das crises vitais e ansiedades existenciais durante o desenvolvimento do adolescente ${ }^{15,21}$, um relacionamento aberto pautado no diálogo e busca de novas formas de relacionamento intra e extra familiar ${ }^{22,23}$, se associaram com maiores níveis de atividade física e menor sedentarismo em adolescentes ${ }^{24}$. Outros fatores familiares associados foram o incentivo para a realização de atividades de lazer que não envolvam exposição à tela ${ }^{25}$ e a promoção de hábitos de atividade física, por meio de prática de atividade física pelos pais, em que há um aumento na probabilidade dos filhos serem ativos quando os pais possuem esse hábito, ou por possibilitarem o acesso dos filhos a equipamentos e a participação conjunta na prática de atividade física ${ }^{23,24,26}$.

Apesar de existirem estudos sobre supervisão parental, sedentarismo e inatividade física, a maioria deles tem representatividade local e foram realizados em países de alta renda ${ }^{27-29}$. Os resultados dos estudos de países de alta renda dificilmente podem ser transpostos para a realidade de países de média renda, como o Brasil ${ }^{30}$. Isso porque países de alta renda diferem dos de média e baixa renda em vários aspectos, dentre os quais destacam-se maiores investimentos em políticas públicas para a promoção de modos de vida ativa, tais como maior disponibilidade de parques e espaços públicos para prática de atividade física e de transporte ativo (ex. ciclovias) ${ }^{31-35}$. Além disso, aspectos culturais, menores níveis de violência e maior segurança percebida, influenciam de forma distinta os processos de supervisão parental e os níveis de atividade física e sedentarismo ${ }^{14}$. Ademais, como a supervisão parental é fator modificável, seria possível atuar para aumentar a atividade física e reduzir o sedentarismo a partir de intervenções de aconselhamento famíliar ${ }^{26}$ que podem ser estratégias de baixo custo, factíveis em países de média e baixa renda.

Nesse sentido, nosso estudo teve o objetivo de avaliar a associação entre características de supervisão parental e comportamentos sedentários e de inatividade física entre adolescentes brasileiros.

\section{Métodos}

Trata-se de um estudo transversal que utilizou dados da Pesquisa Nacional de Saúde do Escolar (PeNSE), realizada em 2015. A seleção da amostra da PeNSE foi baseada no cadastro do Censo Escolar de 2013, formado pelas escolas de ensino fundamental (públicas e privadas) que possuíam turmas do $9^{\circ}$ ano do ensino fundamental. A amostragem foi em conglomerado com estratificação e seleção em múltiplos estágios. Os estratos amostrais foram formados por 53 estratos geográficos, sendo, 26 estratos das capitais de cada unidade da federação mais o Distrito Federal e 26 estratos das unidades da federação com municípios não capitais. Após selecionadas as escolas aleatoriamente, as turmas do $9^{\circ}$ ano foram selecionadas aleatoriamente de forma que escolas com até duas turmas do $9^{\circ}$ ano tiveram uma turma selecionada e escolas com três ou mais tur- 
mas, tiveram duas turmas selecionadas. Em cada turma selecionada, todos os alunos presentes foram convidados a responder ${ }^{36}$.

\section{Coleta de dados}

Os dados foram coletados de abril a setembro de 2015. Os escolares preencheram o questionário em aplicativos de smartphone na sala de aula. No inquérito havia perguntas referentes às condições socioeconômicas; contextos familiares; alimentação; prática de atividade física; uso de cigarro, álcool e outras drogas; comportamento sexual; violência, segurança e acidentes; saúde mental; uso de serviços de saúde, dentre outros.

Demais informações sobre a metodologia da PeNSE estão descritas em um estudo realizado por Oliveira e colaboradores ${ }^{37}$.

\section{Variáveis de interesse}

Os desfechos do presente estudo foram a inatividade física e o sedentarismo. Adotou-se para o cálculo de atividade física a recomendação da Organização Mundial da Saúde (OMS), que considera a somatória do tempo de deslocamento, educação física escolar e atividade física de lazer, sendo classificado como ativo o adolescente que realiza no mínimo 60 minutos diariamente ${ }^{7,23}$. No presente estudo, consideramos como ativos os adolescentes que cumpriam no mínimo 420 minutos de atividade física por semana, que seria o equivalente aos 60 minutos diários durante sete dias na semana. Para chegar a essa categorização foram utilizadas oito variáveis sobre a frequência de realização de atividade física nas três dimensões definidas pela OMS e a duração dessas atividades. O comportamento sedentário foi avaliado a partir da variável "Em um dia de semana comum, quanto tempo você fica sentado assistindo televisão, usando computador, jogando vídeo game, conversando com os amigos ou fazendo outras atividades sentado (a)? (não contar sábado, domingo, feriados e o tempo sentado na escola)". Os adolescentes que passavam 3 horas ou mais sentados foram classificados como sedentários ${ }^{38}$. Embora outros pontos de corte sejam utilizados, revisão de literatura realizada em 2016 mostrou que 3 horas/dia era o mais utilizado para adolescentes. Dessa forma, adotamos o ponto de corte de $3 \mathrm{~h} /$ dia para permitir comparabilidade dos nossos resultados com a literatura sobre o tema ${ }^{5}$.

A supervisão parental que foi definida como a monitoria realizada por pais ou responsáveis do adolescente constituiu nossa exposição principal e foi avaliada a partir de diversas perguntas. Foi avaliada a frequência (nunca, às vezes ou sempre) que o adolescente reportava almoçar ou jantar com os pais/responsáveis, se havia faltado à aula sem permissão dos pais, se os pais sabiam o que o adolescente fazia no tempo livre, se os pais verificavam o dever de casa, se os pais entendiam os problemas e preocupações do adolescente e se os pais mexiam nas coisas do adolescente sem a concordância do mesmo.

Além disso, foram avaliadas as seguintes características sociodemográficas: sexo (masculino/feminino), idade (11 a 13, 14, 15, $\geq 16$ anos), escolaridade materna (ensino fundamental incompleto, ensino fundamental completo, ensino médio completo e ensino superior completo), arranjo familiar (não mora com pai nem mãe, mora com apenas pai ou mãe, mora com ambos os pais), cor da pele (branca, preta, amarela, parda e indígena), dependência administrativa da escola (pública ou privada) e escore de bens e serviços divididos em quartis $(1,2,3,4)$.

Para avaliação do nível socioeconômico foram utilizadas as variáveis escolaridade materna e escore de bens e serviços (EBS). Para a elaboração do EBS foram utilizadas as seguintes variáveis: posse de computador, telefone fixo, celular, carro, acesso à internet e presença de empregada doméstica. Para o cálculo do escore cada item recebeu um peso obtido através do inverso da frequência da posse ou presença do bem seguindo metodologia de Levy et al. ${ }^{39}$. O escore de cada adolescente foi obtido através da soma dos pesos de cada item. Posteriormente a distribuição do escore foi dividida em quartis.

\section{Análises estatísticas}

As variáveis foram inicialmente examinadas por meio de análises descritivas para caracterizar a população de estudo. Em seguida, estimaramse as prevalências de inatividade física e sedentarismo em função de todas as covariáveis do estudo, com o respectivo intervalo de confiança das prevalências.

Houve cerca de $25 \%$ de dados faltantes (missing data) para a variável escolaridade materna. Para lidar com isso, foi realizada a imputação múltipla por equações em cadeias que consiste em uma série de modelos de imputação, sendo que para a variável escolaridade materna utilizou-se o modelo de regressão logística ordinal ${ }^{40}$. Também foram incluídas na imputação outras variáveis com quantidades menores de dados fal- 
tantes para se obter uma informação mais completa e precisa. A partir das informações obtidas com o banco imputado, foram realizadas as análises do presente trabalho.

A obtenção dos Odds Ratios (OR) brutos nas análises de associação de cada variável com cada desfecho foram realizadas por meio da regressão logística simples. Para avaliar a associação entre as variáveis de supervisão parental com o sedentarismo e a inatividade física realizou-se os modelos de regressão logística múltiplos, ajustados para as variáveis sociodemográficas. A partir da revisão da literatura foram selecionadas potenciais variáveis de confusão seguindo o critério epidemiológico de confundimento. Dessa forma, variáveis que se associavam tanto ao desfecho quanto à variável de exposição e não faziam parte da cadeia causal ${ }^{41}$, foram incluídas no modelo final para ajuste.

Todas as análises foram realizadas considerando a ponderação para a estrutura amostral complexa, de modo a representar a população de escolares matriculados e frequentando o $9^{\circ}$ ano do ensino fundamental em 2015.

Foi estabelecido arbitrariamente como ponto de corte para associação estatisticamente significante $\mathrm{o}$ valor de $\mathrm{p}<0,05$. Para avaliar a associação entre as covariáveis ordinais e o desfecho utilizou-se o $p$ de tendência.

\section{Aspectos éticos}

A PeNSE 2015 foi aprovada pela Comissão Nacional de Ética em Pesquisa (Conep) e o acesso ao banco de dados da pesquisa é livre, disponibilizado em sítio da internet pelo IBGE sem informações que possam identificar os sujeitos e as escolas.

\section{Resultados}

Foram avaliados dados de 102.072 escolares. A maioria dos adolescentes tinha idade de 14 anos $(51,0 \%)$, era do sexo feminino $(51,3 \%)$ e morava com ambos os pais (59,3\%). $34,4 \%$ dos adolescentes tinham mães com baixa escolaridade (ensino fundamental incompleto) e $43,1 \%$ se declarou de cor parda $(43,1 \%)$. A maioria dos adolescentes eram sedentários (56,3\%) e inativos $(78,1 \%)$.

Na Tabela 1 são observados os percentuais de sedentarismo e inatividade física, de acordo com as covariáveis. De modo geral, meninas mais jovens eram mais inativas e sedentárias. Quanto ao nível socioeconômico, adolescentes com mães com menor escolaridade eram mais inativos, enquanto aqueles com mães com maior escolaridade eram mais sedentários, tendência semelhante foi observada para os quartis do EBS.

Comparando os modelos brutos e ajustados, observou-se que a maioria das associações permaneceram significativas após o ajuste.

Nos modelos ajustados observamos que características de supervisão parental como os responsáveis sempre estarem cientes das atividades do filho no tempo livre (OR=0,82; IC95\% $=0,74$ $0,91)$, sempre verificarem as atividades escolares $(\mathrm{OR}=0,70$; IC95\% $=0,65-0,76)$ e sempre mexerem nos pertencem pessoais dos filhos sem permissão (OR $=0,78$; IC95\% $=0,72-0,84)$, se associaram a menor odds de inatividade física dos adolescentes, tendo como referência pais que nunca adotavam as referidas atitudes. Essa associação também ocorreu entre adolescentes que relataram sempre faltarem às aulas sem permissão dos responsáveis $(\mathrm{OR}=0,67$; IC95\% $=0,54-0,81)$, comparados aos que relataram nunca fazerem isso, e com o aumento da escolaridade materna $(\mathrm{OR}=0,85 ;$ IC95\%=0,77-0,93) (Tabela 2).

Em relação ao sedentarismo, adolescentes cujos responsáveis sempre estavam cientes das atividades no tempo livre $(\mathrm{OR}=1,38$; $\mathrm{IC} 95 \%=1,27-1,51)$, que sempre faltavam às aulas sem permissão dos responsáveis $(\mathrm{OR}=1,94$; IC95\% $=1,63-2,30)$, sempre tinham seus pertences remexidos sem a sua autorização $(\mathrm{OR}=1,27$; IC95\% $=1,18-1,37)$ e filhos de mães com maior escolaridade materna $(\mathrm{OR}=1,21$; IC $95 \%=1,10$ $1,33)$ tiveram maior odds de serem sedentários, comparados aos adolescentes cujos pais nunca adotavam tais atitudes e aos filhos de mães com menor escolaridade. Por outro lado, menores odds de sedentarismo foram observados entre adolescentes cujos responsáveis sempre verificavam as atividades escolares $(\mathrm{OR}=0,61$; IC $95 \%=0,57-0,65)$ e sempre compreendiam os seus problemas (OR $=0,72$; IC $95 \%=0,67-0,78)$, comparados aos adolescentes cujos pais nunca adotavam essas atitudes (Tabela 3 ).

\section{Discussão}

Os resultados mostram altas prevalências de sedentarismo $(56,3 \%)$ e inatividade física $(78,1 \%)$ entre escolares brasileiros, e que esses comportamentos se associam à supervisão parental. Adolescentes cujos responsáveis sempre verificavam as atividades escolares tiveram menor odds de 
Tabela 1. Distribuição das Características sociodemográficas de acordo com o sedentarismo e inatividade física em adolescentes brasileiros, Pesquisa Nacional de Saúde do Escolar (PeNSE, 2015).

\begin{tabular}{|c|c|c|c|c|}
\hline Características sociodemográficas & Inativos (\%) & IC95\% & Sedentários (\%) & IC95\% \\
\hline \multicolumn{5}{|l|}{ Sexo } \\
\hline Feminino & 85,2 & $84,5-85,8$ & 57,8 & $56,9-58,7$ \\
\hline Masculino & 70,6 & $69,7-71,4$ & 54,8 & $53,8-55,8$ \\
\hline \multicolumn{5}{|l|}{ Idade } \\
\hline 11 a 13 anos & 80,3 & $78.9-81,7$ & 59,2 & $57,6-60,8$ \\
\hline 14 anos & 78,7 & $77,9-79,5$ & 58,6 & $57,7-59,6$ \\
\hline 15 anos & 75,5 & $74,5-76,5$ & 52,8 & $51,4-54,1$ \\
\hline$\geq 16$ anos & 75,9 & $74,6-77,2$ & 47,2 & $45,7-48,7$ \\
\hline \multicolumn{5}{|l|}{ Raça } \\
\hline Branca & 79,2 & $78,4-80,0$ & 59,8 & $58,7-60,9$ \\
\hline Preta & 74,0 & $72,4-75,5$ & 52,7 & $50,9-54,5$ \\
\hline Amarela & 78,2 & $76,0-80,5$ & 59,3 & $56,5-62,0$ \\
\hline Parda & 78,5 & $77,7-79,3$ & 54,3 & $53,4-55,2$ \\
\hline Indígena & 76,0 & $73,6-78,4$ & 55,3 & $52,3-58,3$ \\
\hline \multicolumn{5}{|l|}{ Arranjo Familiar } \\
\hline Não mora com pai nem mãe & 77,4 & $75,4-79,3$ & 50,6 & $48,5-52,6$ \\
\hline Mora apenas com o pai ou mãe & 76,7 & $75,9-77,6$ & 58,8 & $57,7-59,9$ \\
\hline Mora com ambos os pais & 79,0 & $78,2-79,7$ & 55,4 & $54,5-56,3$ \\
\hline \multicolumn{5}{|l|}{ Escolaridade da mãe } \\
\hline Ensino Fundamental incompleto & 79,9 & $79,1-80,8$ & 48,7 & $47,3-50,0$ \\
\hline Ensino Fundamental completo & 79,5 & $78,3-80,8$ & 56,5 & $54,9-58,0$ \\
\hline Ensino médio completo & 77,3 & $76,2-78,3$ & 61,3 & $60,0-62,5$ \\
\hline Superior completo & 76,4 & $75,0-77,7$ & 62,2 & $60,7-63,6$ \\
\hline \multicolumn{5}{|l|}{ Quartis de Bens e Serviços } \\
\hline 1 & 80,3 & $79,4-81,2$ & 40,3 & $39,3-41,4$ \\
\hline 2 & 78,7 & $77,3-78,9$ & 58,5 & $57,5-59,5$ \\
\hline 3 & 77,8 & $76,7-79,0$ & 65,8 & $64,7-66,9$ \\
\hline 4 & 73,3 & $71,5-75,1$ & 60,1 & $58,4-61,7$ \\
\hline \multicolumn{5}{|l|}{ Dependência Administrativa da escola } \\
\hline Pública & 77,6 & $77,0-78,2$ & 54,8 & $54,0-55,6$ \\
\hline Privada & 80,7 & $79,4-82,0$ & 65,2 & $63,4-67,0$ \\
\hline Total & 78,1 & $77,4-78,6$ & 56,3 & $55,6-57,1$ \\
\hline
\end{tabular}

serem sedentários e inativos comparados aos responsáveis que nunca verificavam. Escolares que faltavam à escola sem o conhecimento dos responsáveis, com responsáveis sempre cientes do tempo livre e que sempre mexiam nos pertences pessoais sem permissão dos mesmos, tiveram maior odds de serem sedentários e menor odds de serem inativos. Também foi observado menor odds de comportamento sedentário entre adolescentes cujos responsáveis sempre compreendiam seus problemas.

Os escolares cujos responsáveis sempre verificavam atividades que a escola envia para casa, tiveram 30\% menor odds de serem inativos e $39 \%$ menor odds de serem sedentários, compa- rados àqueles cujos pais nunca verificavam as atividades. Nossos resultados corroboram com estudo realizado com dados de sete países, que mostrou que escolares cujos pais ou responsáveis supervisionavam a realização dos deveres escolares tiveram menor odds de serem inativos e sedentários $^{42}$. Outro estudo também observou que, comparados aos escolares que os pais não verificavam atividades que a escola envia para a casa, adolescentes que recebiam supervisão destas atividades tiveram menor odds de serem inativos e sedentários ${ }^{43}$. Esses resultados sugerem que a supervisão parental pode trazer benefícios para a saúde dos adolescentes. Segundo a literatura, os alunos mais acompanhados são também mais in- 
Tabela 2. Associação entre a supervisão parental e a inatividade física entre escolares brasileiros, Pesquisa Nacional de Saúde do Escolar (PeNSE, 2015).

\begin{tabular}{|c|c|c|c|c|c|c|c|}
\hline \multirow{2}{*}{ Variáveis } & \multirow{2}{*}{$\begin{array}{c}\text { Inativos } \\
(\%)\end{array}$} & \multicolumn{3}{|c|}{ Modelo bruto } & \multicolumn{3}{|c|}{ Modelo ajustado* $^{*}$} \\
\hline & & OR & IC95\% & P-valor & OR & IC95\% & P-valor \\
\hline Supervisão parental & & \multicolumn{6}{|c|}{ Inatividade Física } \\
\hline \multicolumn{8}{|l|}{ Almoça com responsáveis } \\
\hline Nunca & 77,13 & 1,00 & & 0,056 & 1,00 & & 0,353 \\
\hline Às vezes & 80,00 & 1,19 & $1,04-1,35$ & & 1,10 & $0,96-1,26$ & \\
\hline Sempre & 77,67 & 1,03 & $0,91-1,17$ & & 1,09 & $0,96-1,24$ & \\
\hline \multicolumn{8}{|l|}{ Falta sem permissão dos responsáveis } \\
\hline Nunca & 78,88 & 1,00 & & $<0,001$ & 1,00 & & $<0,001$ \\
\hline Às vezes & 76,85 & 0,89 & $0,83-0,96$ & & 0,94 & $0,87-1,01$ & \\
\hline Sempre & 70,18 & 0,63 & $0,51-0,78$ & & 0,67 & $0,54-0,81$ & \\
\hline \multicolumn{8}{|l|}{ Responsáveis cientes do tempo livre } \\
\hline Nunca & 79,42 & 1,00 & & $<0,001$ & 1,00 & & $<0,001$ \\
\hline Às vezes & 79,79 & 1,02 & $0,93-1,13$ & & 0,97 & $0,85-1,08$ & \\
\hline Sempre & 77,54 & 0,89 & $0,82-0,97$ & & 0,82 & $0,74-0,91$ & \\
\hline \multicolumn{8}{|c|}{ Responsáveis verificam atividades escolares } \\
\hline Nunca & 82,07 & 1,00 & & $<0,001$ & 1,00 & & $<0,001$ \\
\hline Às vezes & 79,50 & 0,85 & $0,79-0,91$ & & 0,94 & $0,87-1,01$ & \\
\hline Sempre & 73,47 & 0,60 & $0,56-0,65$ & & 0,70 & $0,65-0,76$ & \\
\hline \multicolumn{8}{|l|}{$\begin{array}{l}\text { Compreensão de problemas pelos } \\
\text { responsáveis }\end{array}$} \\
\hline Nunca & 80,92 & 1,00 & & $<0,001$ & 1,00 & & 0,049 \\
\hline Às vezes & 79,52 & 0,91 & $0,85-0,99$ & & 0,99 & $0,90-1,08$ & \\
\hline Sempre & 76,09 & 0,75 & $0,70-0,81$ & & 0,93 & $0,85-1,01$ & \\
\hline \multicolumn{8}{|c|}{ Responsáveis mexem nos pertences pessoais } \\
\hline Nunca & 79,19 & 1,00 & & $<0,001$ & 1,00 & & $<0,001$ \\
\hline Às vezes & 78,51 & 0,96 & $0,90-1,02$ & & 0,96 & $0,91-1,02$ & \\
\hline Sempre & 73,93 & 0,74 & $0,69-0,81$ & & 0,78 & $0,72-0,84$ & \\
\hline \multicolumn{8}{|l|}{ Escolaridade materna } \\
\hline Ensino Fundamental incompleto & 79,94 & 1,00 & & $<0,001$ & 1,00 & & $<0,001$ \\
\hline Ensino Fundamental completo & 79,55 & 0,98 & $0,89-1,07$ & & 1,02 & $0,93-1,12$ & \\
\hline Ensino médio completo & 77,26 & 0,85 & $0,79-0,92$ & & 0,89 & $0,82-0,97$ & \\
\hline Superior completo & 76,39 & 0,81 & $0,74-0,88$ & & 0,82 & $0,77-0,93$ & \\
\hline
\end{tabular}

centivados pelos pais a praticarem atividades de lazer que não envolvam exposição à tela ${ }^{44}$ e que envolvam a prática de atividade física ${ }^{45}$.

Um estudo com 110 crianças de 9 a 12 anos de uma escola de São Paulo mostrou que pais que se envolvem com maior frequência nas atividades escolares, culturais e de lazer de seus filhos, conseguem demonstrar maior interesse pelas suas atividades de rotina e dessa forma fortalecem os laços familiares ${ }^{46}$.

Escolares cujos responsáveis sempre entendiam seus problemas e preocupações apresentaram 28\% menor odds de serem sedentários, comparados aqueles cujos pais nunca adotavam essa atitude. Talvez isso possa ser explicado pelo fato de haver o fortalecimento na relação entre pais e filhos, tornando-os mais adaptados a novos estilos de vida ${ }^{47}$. Esse resultado está de acordo com a literatura, que mostra que a supervisão parental diminui a probabilidade de sedentarismo ${ }^{42}$.

Adolescentes que sempre faltaram às aulas sem a permissão dos responsáveis tiveram 94\% maior odds de serem sedentários e 33\% menor odds de serem inativos, comparados aos que relataram nunca fazer isso. Estudos mostram que o absenteísmo escolar aumenta as chances de sedentarismo, além do envolvimento em outros comportamentos de risco para a saúde, como 
Tabela 3. Associação entre a supervisão parental e o sedentarismo entre escolares brasileiros, Pesquisa Nacional de Saúde do Escolar (PeNSE, 2015).

\begin{tabular}{|c|c|c|c|c|c|c|c|}
\hline \multirow{2}{*}{ Variáveis } & \multirow{2}{*}{$\begin{array}{c}\text { Sedentários } \\
(\%)\end{array}$} & \multicolumn{3}{|c|}{ Modelo bruto } & \multicolumn{3}{|c|}{ Modelo ajustado* $^{*}$} \\
\hline & & OR & IC95\% & P-valor & OR & IC95\% & P-valor \\
\hline Supervisão parental & & \multicolumn{6}{|c|}{ Sedentarismo } \\
\hline \multicolumn{8}{|l|}{ Almoça com responsáveis } \\
\hline Nunca & 57,94 & 1,00 & & $<0,001$ & 1,00 & & $<0,001$ \\
\hline Às vezes & 62,48 & 1,21 & $1,09-1,33$ & & 1,11 & $0,99-1,23$ & \\
\hline Sempre & 54,50 & 0,87 & $0,79-0,95$ & & 0,94 & $0,85-1,04$ & \\
\hline \multicolumn{8}{|l|}{ Falta sem permissão dos responsáveis } \\
\hline Nunca & 55,05 & 1,00 & & $<0,001$ & 1,00 & & $<0,001$ \\
\hline Às vezes & 59,01 & 1,17 & $1,11-1,24$ & & 1,20 & $1,13-1,27$ & \\
\hline Sempre & 69,44 & 1,85 & $1,59-2,17$ & & 1,94 & $1,63-2,30$ & \\
\hline \multicolumn{8}{|l|}{ Responsáveis cientes do tempo livre } \\
\hline Nunca & 48,89 & 1,00 & & $<0,001$ & 1,00 & & $<0,001$ \\
\hline Às vezes & 57,85 & 1,43 & $1,31-1,56$ & & 1,29 & $1,18-1,42$ & \\
\hline Sempre & 56,96 & 1,38 & $1,29-1,48$ & & 1,38 & $1,27-1,51$ & \\
\hline \multicolumn{8}{|l|}{$\begin{array}{l}\text { Responsáveis verificam atividades } \\
\text { escolares }\end{array}$} \\
\hline Nunca & 62,55 & 1,00 & & $<0,001$ & 1,00 & & $<0,001$ \\
\hline Às vezes & 58,28 & 0,84 & $0,79-0,89$ & & 0,83 & $0,78-0,88$ & \\
\hline Sempre & 48,55 & 0,56 & $0,53-0,60$ & & 0,61 & $0,57-0,65$ & \\
\hline \multicolumn{8}{|l|}{$\begin{array}{l}\text { Compreensão de problemas pelos } \\
\text { responsáveis }\end{array}$} \\
\hline Nunca & 61,62 & 1,00 & & $<0,001$ & 1,00 & & $<0,001$ \\
\hline Às vezes & 58,64 & 0,88 & $0,82-0,94$ & & 0,87 & $0,81-0,93$ & \\
\hline Sempre & 52,10 & 0,68 & $0,63-0,73$ & & 0,72 & $0,67-0,78$ & \\
\hline \multicolumn{8}{|l|}{$\begin{array}{l}\text { Responsáveis mexem nos pertences } \\
\text { pessoais }\end{array}$} \\
\hline Nunca & 52,99 & 1,00 & & $<0,001$ & 1,00 & & $<0,001$ \\
\hline Às vezes & 59,01 & 1,28 & $1,21-1,35$ & & 1,17 & $1,10-1,24$ & \\
\hline Sempre & 59,70 & 1,31 & $1,22-1,41$ & & 1,27 & $1,18-1,37$ & \\
\hline \multicolumn{8}{|l|}{ Escolaridade materna } \\
\hline Ensino Fundamental incompleto & 48,69 & 1,00 & & $<0,001$ & 1,00 & & $<0,001$ \\
\hline Ensino Fundamental completo & 56,46 & 1,37 & $1,26-1,48$ & & 1,18 & $1,08-1,28$ & \\
\hline Ensino médio completo & 61,27 & 1,67 & $1,54-1,80$ & & 1,27 & $1,17-1,39$ & \\
\hline Superior completo & 62,16 & 1,73 & $1,60-1,88$ & & 1,21 & $1,10-1,33$ & \\
\hline
\end{tabular}

${ }^{\star}$ Ajustados pelas variáveis sociodemográficas sexo, idade, raça/cor, arranjo familiar, escolaridade materna e quartis de bens e serviços. P-valor obtido para teste de tendência.

experimentação de álcool e drogas ${ }^{43,48}$. Esses resultados podem estar relacionados ao fato desses adolescentes estarem acompanhados por pares, durante os momentos em que faltam às aulas sem o conhecimento dos pais. Dessa forma, os adolescentes podem tanto se engajar mais em atividades sedentárias (aumentando o sedentarismo) quanto se envolver em esportes ou outras atividades físicas com os pares (o que aumentaria atividade física). Estudo mostrou que adolescentes que saem mais com amigos tendem a ser mais ativos $^{13}$. Devemos considerar que o absenteísmo escolar sem a autorização dos pais pode refletir mais uma característica de desvio de comportamento do adolescente ${ }^{49}$, não necessariamente significando a ausência de supervisão, mas sim um desafio à supervisão parental, visto que quando há menor conectividade na relação pais e filhos ocorre maior absenteísmo escolar ${ }^{50}$. Isso pode explicar os resultados observados e reforçam a necessidade de avaliar sedentarismo e inatividade física separadamente.

Escolares cujos responsáveis sempre sabiam o que os filhos faziam no tempo livre tiveram 18\% 
menos odds de serem inativos, comparados aos que os responsáveis nunca sabiam. Isso corrobora com a literatura pelo fato de que os pais que participam ativamente da vida dos filhos influenciam uma maior aceitação da atividade física por parte da criança ${ }^{51}$. $\mathrm{O}$ incentivo à prática de atividade física é maior em locais onde os pais têm maior controle do tempo livre dos adolescentes e conhecimento do bairro e da vizinhança ${ }^{52,53}$.

No entanto, esse comportamento parental se associou a um odds 38\% maior dos adolescentes serem sedentários, sugerindo que os responsáveis que tem o maior controle do tempo livre dos adolescentes podem também mantê-los por mais tempo sob vigilância dentro de casa levando-os a se engajarem em atividades sedentárias nesse período. Um estudo de coorte indicou que adolescentes cujas mães usam de argumentos como o perigo da vizinhança, as "más companhias", e o baixo desenvolvimento escolar dos adolescentes para privá-los de sair com amigos, tendem a ser mais sedentários, pois apresentam menos possibilidades de lazer e aumentam o tempo em atividades sentadas ${ }^{53}$. Da mesma forma, estudo feito no Canadá apontou que pais mais permissivos quanto aos limites de tempo de exposição à tela aumentam a probabilidade de sedentarismo entre seus filhos. Especialmente se os pais têm os mesmos hábitos de entretenimento no ambiente familiar ${ }^{54}$.

Os escolares cujos responsáveis sempre mexiam nas suas coisas tiveram $22 \%$ menor odds de serem inativos e $27 \%$ maior odds de serem sedentários, comparados aqueles cujos responsáveis nunca adotavam esse comportamento. Não foi encontrado na literatura resultados que expliquem essa associação, especialmente para o aumento do sedentarismo dos adolescentes. No entanto, podemos avaliar esse resultado como uma invasão de privacidade e violação dos direitos dos adolescentes por parte dos pais, podendo apontar uma relação de desconfiança no ambiente familiar e de falta de reciprocidade ${ }^{55}$. Ademais, um estudo aponta que é dever dos pais não mexer nas coisas dos filhos sem sua permissão para que haja uma relação de respeito mútuo no ambiente familiar auxiliando assim na construção da autonomia moral do adolescente ${ }^{45}$.

Escolares com mães com maior escolaridade tiveram $15 \%$ menor odds de serem inativos e $21 \%$ maior odds de serem sedentários, comparados aos filhos de mães com menor escolaridade. Estudo avaliando a inatividade física também encontrou uma relação inversa entre a inatividade e o aumento da escolaridade materna apontando que somente $3,5 \%$ dos adolescentes com mães com ensino superior completo eram inativos ${ }^{56}$. Pode contribuir para a explicação desse resultado o fato de a escolaridade materna ser proxy de ren$\mathrm{da}$, sugerindo que adolescentes com mães mais escolarizadas tem maiores condições socioeconômicas e por sua vez têm mais acesso a atividade física no tempo de lazer ${ }^{57}$. Em contrapartida, a condição socioeconômica favorece o acesso aos recursos tecnológicos o que contribui para o aumento do sedentarismo ${ }^{58}$.

Os resultados do presente estudo devem ser analisados considerando algumas limitações. A primeira limitação refere-se à forma como o sedentarismo e a inatividade física foram avaliados, a partir do auto-relato e utilizando instrumentos não validados. Essa forma indireta de mensurar os comportamentos está sujeita a erros de informação, seja por viés de memória ou por desejabilidade social, o que implicaria em subestimação dos comportamentos não saudáveis. Esses erros são classificados como vieses de informação não diferencial, e tendem a afetar as estimativas de forma a reduzir a magnitude das associações encontradas entre as exposições e os desfechos do presente estudo, e são considerados erros conservadores $^{59}$. Apesar da imprecisão das medidas feitas por questionários, pesquisas com a dimensão populacional da PeNSE não são compatíveis com a realização de estimativas diretas de atividade física ou mesmo a aplicação de instrumentos completos para avaliação desses comportamentos, mas servem como importante instrumento para explorar temas de interesse para a saúde pública. Em que pese essa limitação, outros estudos utilizando instrumentos validados encontraram resultados semelhantes aos do nosso estudo ${ }^{43,60-62}$. Além disso, a supervisão parental foi relatada a partir da percepção dos alunos. Nesse sentido, a supervisão parental aplicada no ambiente familiar poderia ser percebida de forma diferente se fosse obtida a partir do relato dos pais. De qualquer forma, as duas formas se baseariam na percepção da supervisão, porém, a partir de diferentes perspectivas.

Embora as análises tenham sido ajustadas para as principais variáveis de confusão identificadas na literatura ${ }^{26,56,633,64}$, a PeNSE não mensurou a atividade física e o sedentarismo dos pais, que poderia ser uma variável de confusão importante para as análises. Além disso, como todos os estudos observacionais, nosso estudo não pode excluir a possibilidade de que parte das associações encontradas se deva a confundimento residual. 
Outro ponto importante, é que o estudo aborda somente alunos que estão no ambiente escolar, não abrangendo os faltosos, no dia de aplicação, e os que não estão matriculados em escolas. Apesar disso, no Brasil, a cobertura escolar é elevada chegando a $98,6 \%$ e $85 \%$ entre adolescentes de 6 a 14 e de 15 a 17 anos de idade respectivamente, o que sustenta a representatividade da $\operatorname{amostra}^{65}$.

Por se tratar de um estudo transversal, não é possível identificar a direção da associação encontrada, impedindo que se estabeleça relações causais $^{37}$. Entretanto, parece razoável supor que é a supervisão parental que influencia nos hábitos dos adolescentes e não o contrário.

Apesar das limitações o presente estudo também apresenta pontos positivos. A representatividade nacional com amostra que abrange todas as regiões do Brasil é sua principal vantagem. Especialmente em um país com dimensões continentais e tamanha diversidade, resultados de estudos locais podem não refletir a realidade nacional. Além disso, o estudo teve alta taxa de resposta com poucas recusas e perdas, o que reduz a possibilidade de viés de seleção.

A PeNSE foi a primeira base de dados nacionais a trazer dados referente a atividade física em adolescentes, revelando a sua importância para monitorar comportamentos relacionado ao sedentarismo e a inatividade física ${ }^{56}$. Esse estudo pode ser utilizado para auxiliar a elaboração de políticas públicas e contribuir para a integração da saúde em outros setores.

Nossos resultados sugerem que menores níveis de inatividade física e de sedentarismo são observados entre adolescentes com maior supervisão parental. Para, além disso, a inatividade física e o sedentarismo podem se apresentar inversamente relacionados nos adolescentes sugerindo que a influência parental no aumento da prática de atividade física não necessariamente diminui os comportamentos sedentários ou vice e versa. Outros estudos confirmam essa hipótese demonstrando que o sedentarismo é elevado entre os adolescentes independente do nível de atividade física praticada e que a diminuição da inatividade física não diminui comportamentos sedentários ${ }^{66,67}$.

\section{Considerações finais}

Conclui-se que maior supervisão parental se associa a menor chance de comportamento sedentário e de inatividade física em adolescentes. É importante compreender que a supervisão parental pode ter efeitos opostos nesses comportamentos, já que o sedentário e inatividade física possuem determinantes diferentes. As ações para mitigar a inatividade e o sedentarismo devem abranger o contexto familiar e o ambiente em que o adolescente está inserido, como forma de serem mais efetivas.

\section{Colaboradores}

CP Santana, HAS Nunes e CM Azeredo participaram igualmente da concepção do estudo, análise dos dados e redação final do artigo. AN Silva contribuiu com a análise de dados, redação e revisão do manuscrito. Todos os autores aprovaram a versão final do manuscrito. 


\section{Referências}

1. Rezende LFM, Sá TH, Mielke GI, Viscondi JYK, ReyLópez JP, Garcia LMT. All-Cause Mortality Attributable to Sitting Time: Analysis of 54 Countries Worldwide. Am J Prev Med 2016; 51(2):253-263.

2. Lee I-M, Shiroma EJ, Lobelo F, Puska P, Blair SN, Katzmarzyk PT. Impact of Physical Inactivity on the World's Major Non-Communicable Diseases. Lancet 2012; 380(9838):219-229.

3. Meneguci J, Santos DAT, Silva RB, Santos RG, Sasaki JE, Tribess S, Damião R, Júnior JSV. Comportamento sedentário: conceito, implicações fisiológicas e os procedimentos de avaliação. Motricidade 2015; 11(1):160-174.

4. Sedentary Behaviour Research Network. Letter to the Editor: Standardized use of the terms "sedentary" and "sedentary behaviours". Appl Physiol Nutr Metab 2012; 37(3):540-542.

5. Carson V, Hunter S, Kuzik N, Gray CE, Poitras VJ, Chaput J-P, Saunders TJ, Katzmarzyk PT, Okely AD, Gorber SC, Kho ME, Sampson M, Lee H, Tremblay MS. Systematic review of sedentary behaviour and health indicators in school-aged children and youth: an update. Appl Physiol Nutr Metab 2016; 41 (6 (Supl. 3)):240-265.

6. Santaliestra-Pasías AM, Rey-López JP, Aznar LAM, others. Obesity and sedentarism in children and adolescents: what should be bone? Nutr Hosp 2013; 28(Supl. 5):99-104.

7. World Health Organization (WHO). Physical activity [página na Internet]. Disponível em: http://www.who. int/news-room/fact-sheets/detail/physical-activity

8. Lewis BA, Napolitano MA, Buman MP, Williams DM, Nigg CR. Future directions in physical activity intervention research: expanding our focus to sedentary behaviors, technology, and dissemination. J Behav Med 2017; 40(1):112-126.

9. Van Der Ploeg HP, Hillsdon M. Is sedentary behaviour just physical inactivity by another name? Int J Behav Nutr Phys Act 2017; 14(1):142.

10. Rezende LFM, Azeredo CM, Canella DS, Claro RM, Castro IRR, Levy RB, Luíz OC. Sociodemographic and behavioral factors associated with physical activity in Brazilian adolescents. BMC Public Health 2014; 14(1):485.

11. Lucena JMS, Cheng LA, Cavalcante TLM, Silva VA, Farias Júnior JC. Prevalência de tempo excessivo de tela e fatores associados em adolescentes. Rev Paul Pediatr 2015; 33(4):407-414.

12. Fundo das Nações Unidas para a Infância (Unicef). Situação Mundial da Infância 2011. Adolescência: Uma fase de oportunidades. Nova York: Unicef; 2011.

13. Lisboa T, Silva WR, Alexandre JM, Beltrame TS. Social support of family and friends for the practice of physical activity of adolescents: a systematic review. Cad Saúde Coletiva 2018; 26(4):351-359.

14. Patton GC, Sawyer SM, Santelli JS, Ross DA, Afifi R, Allen NB, Arora M, Azzopardi P, Baldwin W, Kakuma R, Kennedy E, Mahon J, McGovern T, Mokdad AH, Patel V, Petroni S, Reavley N, Taiwo K, Waldfogel J, Wickremarathne D, Barroso C, Bhutta Z, Fatusi AO, Mattoo A, Diers J, Fang J, Ferguson J, Ssewamala F, Viner RM. Our future: a Lancet commission on adolescent health and wellbeing. Lancet Lond Engl 2016; 387(10036):2423-2478.
15. Schneider EM, Wilson DK, Kitzman-Ulrich H, George SMS, Alia KA. The Associations of Parenting Factors with Adolescent Body Mass Index in an Underserved Population. J Obes 2013; 715618.

16. Nunes LA. A influência dos estilos parentais na obesidade infantil. Rev Psicol 2011; 1(1):37-46.

17. O'Connor TM, Chen T-A, Baranowski J, Thompson D, Baranowski T. Physical Activity and Screen-MediaRelated Parenting Practices Have Different Associations with Children's Objectively Measured Physical Activity. Child Obes 2013; 9(5):446-453.

18. Prado CV, Lima AV, Fermino RC, Añez CRR, Reis RS. Apoio social e prática de atividade física em adolescentes da rede pública de ensino: qual a importância da família e dos amigos? Cad Saúde Pública 2014; 30(4):827-838.

19. Fermino RC, Rech CR, Hino AAF, Rodriguez Añez $\mathrm{CR}$, Reis RS. Atividade física e fatores associados em adolescentes do ensino médio de Curitiba, Brasil. Rev Saúde Pública 2010; 44(6):986-995.

20. Haines J, Rifas-Shiman SL, Horton NJ, Kleinman K, Bauer KW, Davison KK, Walton K, Austin SB, Field AE, Gillman MW. Family functioning and quality of parent-adolescent relationship: cross-sectional associations with adolescent weight-related behaviors and weight status. Int J Behav Nutr Phys Act 2016; 13:68.

21. Pratta EMM, Santos MA. Família e adolescência: a influência do contexto familiar no desenvolvimento psicológico de seus membros. Psicol Estudo 2007; 12(2):247-256.

22. Centers for Disease Control and Prevention (CDC). Parent Engagement: Strategies for Involving Parents in School Health. Atlanta: U.S. Departament of Health and Human Services. 2012.

23. U.S. Department of Health and Human Services. 2008 Physical Activity Guidelines for Americans [Internet]. 2008 [acessado 2018 Nov 17]. Disponível em: https:// health.gov/paguidelines/2008/

24. Rhodes RE, Kaos MD, Beauchamp MR, Bursick SK, Latimer-Cheung AE, Hernandez H, Warburton DER, Ye Z, Nicholas Graham TC. Effects of home-based exergaming on child social cognition and subsequent prediction of behavior. Scand J Med Sci Sports 2018; 28(10):2234-2242.

25. Orehek E, Ferrer R. Parent Instrumentality for Adolescent Eating and Activity. Ann Behav Med 2019; 53(7):652-664.

26. Seabra AF, Denisa M. Mendonça, Thomis MA, Maia JA. Determinantes biológicos e sócio-culturais associados à prática de atividade física de adolescentes. Cad Saúde Pública 2008; 24(4):721-736.

27. Henriksen PW, Ingholt L, Rasmussen M, Holstein BE. Physical activity among adolescents: The role of various kinds of parental support. Scand J Med Sci Sports 2016; 26(8):927-932.

28. Loprinzi PD, Cardinal BJ, Loprinzi KL, Lee H. Benefits and Environmental Determinants of Physical Activity in Children and Adolescents. Obes Facts 2012; 5(4):597-610.

29. Xu H, Wen LM, Rissel C. Associations of Parental Influences with Physical Activity and Screen Time among Young Children: A Systematic Review. J Obes $2015 ; 546925$. 
30. The World Bank. Brasil [Internet]. 2008 [acessado 2018 Nov 17]. Disponível em: https://data.worldbank. org $/$ country/brazil? locale $=\mathrm{pt}$

31. Atkinson K, Lowe S, Moore S. Human development, occupational structure and physical inactivity among 47 low and middle income countries. Prev Med Rep 2015; 3:40-45.

32. Klepac Pogrmilovic B, O'Sullivan G, Milton K, Biddle SJH, Bauman A, Bull F, Kahlmeier S, Prantts M, Pedisici Z. Int J Behav Nutr Phys Act 2018; 15(1):123.

33. Koyanagi A, Stubbs B, Vancampfort D. Correlates of sedentary behavior in the general population: A cross-sectional study using nationally representative data from six low- and middle-income countries. PLoS ONE 2018; 13(8):e0202222.

34. Mielke GI, Brown WJ, Nunes BP, Silva ICM, Hallal PC. Socioeconomic Correlates of Sedentary Behavior in Adolescents: Systematic Review and Meta-Analysis. Sports Med Auckl Nz 2017; 47(1):61-75.

35. Ng SW, Popkin B. Time Use and Physical Activity: A Shift Away from Movement across the Globe. Obes Rev 2012; 13(8):659-680.

36. Brasil. Instituto Brasileiro de Geografia e Estatística (IBGE). Ministério da Saúde (MS). Pesquisa nacional de saúde do escolar, 2015. Rio de Janeiro: IBGE; 2016.

37. Oliveira MM, Campos MO, Andreazzi MAR, Malta DC. Características da Pesquisa Nacional de Saúde do Escolar - PeNSE. Epidemiol Serv Saúde 2017; 26(3):605-616.

38. Guerra PH, Farias Júnior JC, Florindo AA. Sedentary behavior in Brazilian children and adolescents: a systematic review. Rev Saúde Pública 2016; 50(9):15.

39. Levy RB, Castro IRR, Cardoso LO, Tavares LF, Sardinha LMV, Gomes FS, Costa AWN. Consumo e comportamento alimentar entre adolescentes brasileiros: Pesquisa Nacional de Saúde do Escolar (PeNSE), 2009. Cien Saude Colet 2010; 15(Supl. 2):3085-3097.

40. Royston P, White I. Multiple Imputation by Chained Equations (MICE): Implementation in Stata. J Stat Softw 2011; 45(4):20.

41. Rothman KJ, Greenland S. Precision and validity in epidemiologic studies. In: Rothman KJ, Greenland S, organizadores. Modern epidemiology. $2^{\mathrm{a}}$ ed. Philadelphia: Lippincott, Williams \& WIlkins; 1998.

42. Peltzer K, Pengpid S. Leisure Time Physical Inactivity and Sedentary Behaviour and Lifestyle Correlates among Students Aged 13-15 in the Association of Southeast Asian Nations (ASEAN) Member States, 2007-2013. Int J Environ Res Public Health 2016;13(2):217.

43. El-ammari A, El kazdouh H, Bouftini S, El fakir S, El achhab Y. Level and potential social-ecological factors associated with physical inactivity and sedentary behavior among Moroccan school-age adolescents: a cross-sectional study. Environ Health Prev Med 2017; 22(1):47.

44. Biddle SJH, Petrolini I, Pearson N. Interventions designed to reduce sedentary behaviours in young people: a review of reviews. Br J Sports Med 2014; 48(3):182-186.

45. Weber LND, Prado PM, Viezzer AP, Brandenburg OJ. Identificação de estilos parentais: o ponto de vista dos pais e dos filhos. Psicol Reflex Crítica 2004; 17(3):323331.
46. Cia F, Pamplin RCO, Williams LCA. O impacto do envolvimento parental no desempenho acadêmico de crianças escolares. Psicol Estudo 2008; 13(2):351-360.

47. Rozemberg L, Avanci J, Schenker M, Pires T. Resiliência, gênero e família na adolescência. Cien Saude Colet 2014; 19(3):673-684.

48. Malta DC, Machado ÍE, Felisbino-Mendes MS, Prado RR, Pinto AMS, Oliveira-Campos M, Souza MFM, Assunção AA. Uso de substâncias psicoativas em adolescentes brasileiros e fatores associados: Pesquisa Nacional de Saúde dos Escolares, 2015. Rev Bras Epidemiol 2018; 21(Supl. 1):e18000421.

49. Kearney CA. An Interdisciplinary Model of School Absenteeism in Youth to Inform Professional Practice and Public Policy. Educ Psychol Rev 2008; 20(3):257282.

50. AlMakadma AS, Ramisetty-Mikler S. Student, school, parent connectedness, and school risk behaviors of adolescents in Saudi Arabia. Int J Pediatr Adolesc Med 2015; 2(3):128-135.

51. Mondin EMC. Práticas educativas parentais e seus efeitos na criação dos filhos. Psicol Argum 2008; 26(54):233-244.

52. Condessa LA, Chaves OC, Silva FM, Malta DC, Caiaffa WT. Sociocultural factors related to the physical activity in boys and girls: PeNSE 2012. Rev Saúde Pública 2019; 53:25.

53. Gonçalves H, Hallal PC, Amorim TC, Araújo CL, Menezes A. Fatores socioculturais e nível de atividade física no início da adolescência. Rev Panam Salud Pública 2007; 22(4):246-253.

54. He M, Piché L, Beynon C, Harris S. Screen-related Sedentary Behaviors: Children's and Parents' Attitudes, Motivations, and Practices. J Nutr Educ Behav 2010; 42(1):17-25.

55. Souza APL, Lauda BV, Koller SH. Opiniões e vivências de adolescentes acerca dos direitos ao respeito e privacidade e à proteção contra a violência física no âmbito familiar. Psicol Soc 2014; 26(2):397-409.

56. Hallal PC, Knuth AG, Cruz DKA, Mendes MI, Malta DC. Prática de atividade física em adolescentes brasileiros. Cien Saude Colet 2010; 15(Supl. 2):3035-3042.

57. Ramos L. Desigualdade de rendimentos do trabalho no Brasil no período pós-real. Mercado de Trabalho 2006; 30:21-28.

58. Oliveira TC, Silva AAM, Santos CJN, Silva JS, Conceição SIO. Atividade física e sedentarismo em escolares da rede pública e privada de ensino em São Luís. Rev Saúde Pública 2010; 44(6):996-1004.

59. Szklo M, Nieto FJ. Epidemiology: beyond the basics. Sudbury: Jones and Bartlett Publishers; 2007.

60. Laird Y, Fawkner S, Kelly P, McNamee L, Niven A. The role of social support on physical activity behaviour in adolescent girls: a systematic review and meta-analysis. Int J Behav Nutr Phys Act 2016; $13: 79$.

61. Rushovich BR, Voorhees CC, Davis C, Neumark-Sztainer D, Pfeiffer KA, Elder JP, Going S, Marino VG. The relationship between unsupervised time after school and physical activity in adolescent girls. Int $J$ Behav Nutr Phys Act 2006; 3:20.

62. Trost SG, McDonald S, Cohen A. Measurement of General and Specific Approaches to Physical Activity Parenting: A Systematic Review. Child Obes 2013; 9(Supl. 1):S40-S50. 
63. Ferreira RW, Varela AR, Monteiro LZ, Häfele CA, Santos SJ, Wendt A, Silva ICM. Desigualdades sociodemográficas na prática de atividade física de lazer e deslocamento ativo para a escola em adolescentes: Pesquisa Nacional de Saúde do Escolar (PeNSE 2009, 2012 e 2015). Cad Saúde Pública 2018; 34(4):e00037917.

64. Wells L, Nermo M, Östberg V. Physical Inactivity from Adolescence to Young Adulthood: The Relevance of Various Dimensions of Inequality in a Swedish Longitudinal Sample. Health Educ Behav 2017; 44(3):376384.

65. Brasil. Instituto Brasileiro de Geografia e Estatística (IBGE). Pesquisa nacional por amostra de domicílios: sintese de indicadores 2015. Rio de Janeiro: IBGE; 2016.

66. Fernandes RA, Freitas Júnior IF, Cardoso JR, Vaz Ronque ER, Loch MR, Oliveira AR. Association between regular participation in sports and leisure time behaviors in Brazilian adolescents: A cross-sectional study. BMC Public Health 2008; 8:329.

67. Silva DAS, Tremblay MS, Gonçalves ECA, Silva RJ, Santos RD. Television Time among Brazilian Adolescents: Correlated Factors are Different between Boys and Girls. Sci World J 2014; e794539.

Artigo apresentado em 17/11/2018

Aprovado em 24/07/2019

Versão final apresentada em 26/07/2019

Editores chefes: Romeu Gomes, Antônio Augusto Moura da Silva 